Anderson, William Jr, DG Evans (2007) On the interpretation of recharge estimates from steady-state model calibrations, Ground Water 45(4):499-505, doi:10.111/j.1745-6584.2007.00312.x. Version of record available from Wiley [ISSN: 1431-2174], [DOI: 10.111/j.1745-6584.2007.00312.x]

\title{
On the interpretation of recharge estimates from steady-state model calibrations
}

\begin{abstract}
Ground water recharge is often estimated through the calibration of ground water flow models. We examine the nature of calibration errors by considering some simple mathematical and numerical calculations. From these calculations, we conclude that calibrating a steady-state ground water flow model to water level extremes yields estimates of recharge that have the same value as the time-varying recharge at the time the water levels are measured. These recharge values, however, are a subdued version of the actual transient recharge signal. In addition, calibrating a steady-state ground water flow model to data collected during periods of rising water levels will produce recharge values that underestimate the actual transient recharge. Similarly, calibrating during periods of falling water levels will overestimate the actual transient recharge. We also demonstrate that average water levels can be used to estimate the actual average recharge rate provided that water level data have been collected for a sufficient amount of time.
\end{abstract}

\section{Introduction}

Ground water recharge (or water table accretion) is typically one of the more difficult hydrologic parameters to estimate: There is no simple way to measure recharge directly, and it is difficult to estimate using a water budget because the requisite parameters, such as evapotranspiration and soil moisture, are spatially variable and tend to have large measurement errors. In addition, there may be other factors causing water table fluctuations such as pumping and variations in boundary conditions (e.g., stream-level and tidal fluctuations).

These problems notwithstanding, accurate recharge estimates are often necessary to accurately simulate ground water flow, especially in water table aquifers. Therefore, hydrogeologists typically use recharge as a calibration variable that is systematically adjusted during model calibration to provide model results that adequately match historical water level data (e.g., see discussion in Anderson and Woessner 1992). Using recharge as a calibration variable has some significant drawbacks; namely, computed water table elevations tend to be sensitive to recharge estimates and other calibration variables, particularly hydraulic conductivity, tend to covary with recharge. Consequently, independent and accurate estimates of hydraulic conductivity are essential in order to accurately estimate recharge. Perhaps the biggest drawback of using recharge as a calibration variable is that water table fluctuations occur over timescales ranging from hours (for eventdriven fluctuations) to months (for seasonal fluctuations) to years (for interannual fluctuations such as the El NiñoSouthern Oscillation and the Pacific Decadal Oscillation; e.g., see Hanson et al. 2004). Therefore, if recharge is to be a calibration variable for a time-varying model, water levels must be measured with high frequency and for a long duration, which is often unfeasible.

In order to ameliorate this sampling problem, hydrogeologists sometimes calibrate steady-state ground water flow models to synoptic water level data. This approach may be appropriate depending on the objectives of the modeling exercise. There are many examples in the literature that depict the calibration of ground water flow models to steady-state recharge rates (see, for example, 
Anderson et al. 2000; Ayers and Vacher 1983; and Kim et al. 1999). If, however, one goal of the model is to estimate recharge, then significant errors can be introduced by calibrating to a steady-state ground water flow model. In this article, we examine the accuracy of recharge estimates made by calibrating ground water flow models to steady-state recharge. We employ complementary approaches: we use simple mathematical arguments to evaluate the distinction between time-varying and steadystate recharge estimates, and then we illustrate these inferences with numerical experiments that simulate realistic field and sampling situations.

\section{Mathematical Considerations}

In this section, we use mathematical arguments to compare recharge rates estimated by calibrating a steadystate model to the transient recharge that actually produced the observed water level at any time. We also consider the situation in which steady-state recharge rates are calibrated to average water level data rather than to water levels collected at a specific time.

\section{Calibration of a Steady-State Model to Transient Water Level Data}

To begin, consider the approximate ground water flow equation for a water table aquifer. The water table elevation in a homogeneous isotropic water table aquifer is well approximated by the nonlinear Boussinesq equation:

$$
\frac{K}{2} \nabla^{2} h^{2}+w(\vec{x}, t)=S_{\mathrm{y}} \frac{\partial h}{\partial t}
$$

where $K$ is the hydraulic conductivity, $S_{\mathrm{y}}$ is the specific yield, $h$ is the water table elevation, and $w(\vec{x}, t)$ is the recharge, which might vary spatially and temporally (e.g., see Wang and Anderson 1982). Under steady-state conditions, Equation 1 becomes the following:

$$
K \nabla^{2} h^{2}+2 w(\vec{x})=K \nabla^{2} h^{2}+2 w_{\mathrm{SS}}(\vec{x})=0
$$

where the subscript SS has been added to indicate that $w_{\mathrm{SS}}(\vec{x})$ is the steady-state recharge required to produce the water table elevation $h$. Most water table aquifers will never reach a condition of steady state in the sense that water levels will be unchanging in response to constant recharge. Equation 2, however, will be true at the instant when the water table is at a maximum or minimum (because $\partial h / \partial t=0$ at extremes). Therefore,

$$
w_{\mathrm{SS}}(\vec{x})=w(\vec{x}, t)
$$

when the fluctuating water table is at a maximum or minimum. This means that if a steady-state ground water flow model is calibrated to maximum or minimum water levels, the inferred steady-state recharge is equal to the instantaneous recharge at the time the water levels were measured.

If, as is most often the case, water levels are rising or falling at the time they are measured (i.e., not a maximum or minimum), then the inferred steady-state recharge will not equal the actual recharge. To investigate this, we need to only examine the integrated continuity equation for ground water flow. Specifically, if $w(\vec{x}, t)$ is the rate of water entering the aquifer by water table accretion over an area $A$, and $Q(\vec{x}, t)$ is the net rate of water leaving the aquifer through discharge, then:

$$
w(\vec{x}, t) A-Q(\vec{x}, t)=\frac{\mathrm{d} V(\vec{x})}{\mathrm{d} t}
$$

where $V$ is the volume of water in the aquifer. When water levels are falling, the right-hand side of Equation 4 is negative, indicating that $w A<Q$. If, however, we model the water level assuming steady-state conditions, then we implicitly assume that $w A=Q$, thereby overestimating instantaneous recharge. Similarly, steady-state calibration during periods of rising water level will underestimate instantaneous recharge.

By combining the results of the previous two paragraphs, we can immediately conclude that extreme values of modeled steady-state recharge will be less than the actual recharge extremes. That is,

$$
\left|w_{\mathrm{SS}}(\vec{x})\right| \leq|w(\vec{x}, t)|
$$

\section{Analytic Calculations of Recharge Estimated from the Average Water Level}

In this section, we present analytical calculations to show that recharge estimates based on average water table elevation provide estimates of the actual average recharge over the period of time for which the water level was observed. We do this by deriving an analytical solution to the ground water flow equation when recharge is an arbitrary function of time. We then average this solution over time and "calibrate" a steady-state ground water flow model to this average recharge value. The value of steady-state recharge that we calculate in this way is then compared to the recharge value we calculate by averaging the input recharge function and is shown to be equivalent.

To derive an analytical solution to the ground water flow equation, consider the aquifer illustrated in Figure 1, where the ground water divide is located at $x=0$ and the head is fixed at a distance $x=L$ from the divide. The

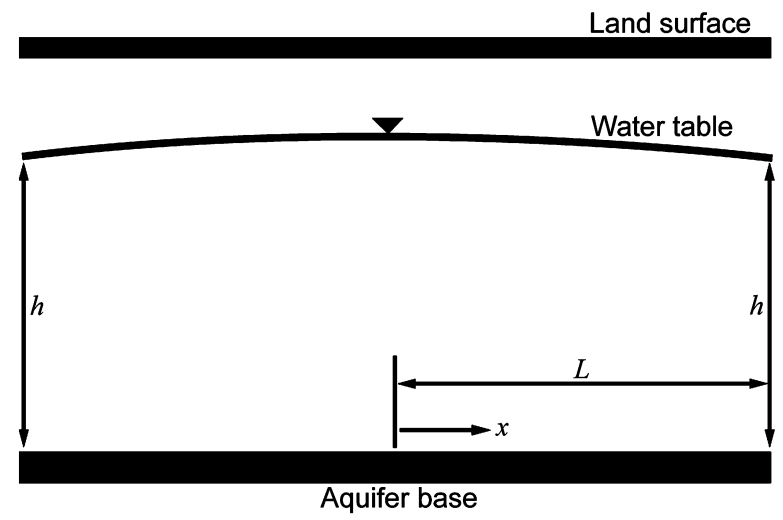

Figure 1. The conceptual model of the one-dimensional aquifer used in the numerical experiments. 
linearized form of the ground water flow equation in an unconfined aquifer is as follows:

$$
\frac{\partial^{2} h}{\partial x^{2}}=\frac{S_{\mathrm{y}}}{T} \frac{\partial h}{\partial t}-\frac{w(t)}{T}
$$

where $T=h_{0} K, h_{0}$ is the initial thickness of the aquifer and $w(t)$ is the recharge, which varies temporally but not spatially.

To solve Equation 6, we write it in dimensionless form using the following substitutions:

$$
\begin{aligned}
h=h^{*} L, x=x^{*} L, t=t^{*} \frac{S_{\mathrm{y}} L^{2}}{T}, \\
A=A^{*} \frac{T}{L}, h_{0}=h_{0}^{*} \frac{T}{S_{\mathrm{y}} L^{2}}, \text { and } w=w^{*} \frac{T}{L}
\end{aligned}
$$

The starred variables are dimensionless. These substitutions give the governing equation in dimensionless form as:

$$
\frac{\partial^{2} h^{*}}{\partial x^{* 2}}=\frac{\partial h^{*}}{\partial t^{*}}-w^{*}
$$

Taking the Laplace transform of both sides of Equation 8, in which we let $\bar{h}$ be the transform of $h, s$ be the Laplace parameter, and $L\left\{w^{*}\right\}$ be the Laplace transform of $w^{*}$, yields a solution in Laplace space of:

$$
\bar{h}\left(x^{*}, s\right)=\frac{1}{s} L\left\{w^{*}\right\}\left[1-\frac{\cosh (\sqrt{s} x)}{\cosh (\sqrt{s})}\right]
$$

In general, it would be quite difficult to find the inverse transform of Equation 9. However, the term in square brackets simplifies nicely for small $s$ (i.e., large time). Simplifying the term in brackets, expanding the result as a series around $s$, and dropping terms of order $s^{2}$ and higher give the following:

$$
\left[1-\frac{\cosh (\sqrt{s} x)}{\cosh (\sqrt{s})}\right] \approx \frac{1}{2} s\left(x^{2}-1\right)
$$

The approximation in Equation 10 applies provided $s \ll 1$, or $1 / t^{*} \ll 1$, or, in dimensional form, when:

$$
t \gg S_{\mathrm{y}} L^{2} / T
$$

By simplifying Equation 9 with the parameter in Equation 11, taking the inverse transform, and returning to dimensional form, we get the following:

$$
h(x, t)=\frac{1}{2 T}\left(L^{2}-x^{2}\right) w(t)
$$

We will use Equation 12 to calculate the average head, $\langle h\rangle$, over some period of time $\hat{t}$ as is shown by:

$$
\langle h\rangle=\frac{1}{\hat{t}} \int_{0}^{\hat{t}} \frac{1}{2 T}\left(L^{2}-x^{2}\right) w(t) \mathrm{d} t
$$

We now turn to the steady-state problem with constant recharge. That is,

$$
\frac{\partial^{2} h_{\mathrm{SS}}}{\partial x^{2}}=-\frac{w_{\mathrm{SS}}}{T}
$$

the solution to which is the following:

$$
h_{\mathrm{SS}}(x)=\frac{w_{\mathrm{SS}}}{2 T}\left(L^{2}-x^{2}\right)
$$

We can calibrate this steady-state ground water flow model to the average head by setting $h_{\mathrm{SS}}=\langle h\rangle$ and solving for $w_{\text {Ss }}$. Using Equation 13 in Equation 15, we find that the steady-state recharge value is given by:

$$
w_{\mathrm{SS}}=\frac{1}{\hat{t}} \int_{0}^{\hat{t}} w(t) \mathrm{d} t=\langle w\rangle
$$

This demonstrates that a constant recharge rate calculated by calibrating a steady-state model to average water table elevation is equal to the average recharge rate calculated over the sampling period.

\section{Discussion}

Several other studies have suggested expressions similar to those shown in Equation 11. Gelhar (1974) analyzes the response of one-dimensional phreatic aquifers to water table accretion. Analyzing a linear Dupuit aquifer, Gelhar (1974) found that the aquifer response time involved the expression $S L^{2} / 3 T$. Zhang and Li (2005), in describing the aquifer response time of Gelhar (1974), claim that recharge is a white noise process when this expression is much greater than 1 . The seminal paper on periodic forcings by Townley (1995), as summarized by Haitjema (1995) and Swanson and Bahr (2004), includes the expression $S L^{2} / T P$, where $P$ represents the period of the oscillation. Again, as in the paper of Zhang and $\mathrm{Li}$ (2005), a value of 1 is a transition point. Aquifer outflow is approximately equal to the steady-state solution and also in phase with that solution when the expression is much less than 1; however, when the expression is much greater than 1, steady-state solutions do not represent the transient system well and are out of phase (Haitjema 1995). Similar diffusive length-scale expressions may also be found in Manga (1996, 1997).

The results of our derivation, and the dimensionless expressions of Gelhar (1974), Townley (1995), Haitjema (1995), and others, have serious implications for those ground water modelers who wish to use time-averaged water levels as a calibration variable in steady-state simulations. Because hydraulic conductivity and therefore transmissivity are often known with confidence only within an order of magnitude, especially in aquifers for which we have limited data, the time span of water level data collection required to accurately estimate average water levels may vary over 2 orders of magnitude (Haitjema 1995). For example, if we have overestimated the actual hydraulic conductivity by an order of magnitude, the calculated monitoring time required to actually achieve the average water level will increase by an order of magnitude; conversely, if we have underestimated the actual hydraulic conductivity by an order of magnitude, the calculated monitoring time required to actually achieve the average water level will decrease by an order of magnitude. 
The simple mathematical results described in this section have important implications with respect to using recharge as a calibration variable in ground water flow models. In the next section, we illustrate these results by estimating recharge values through model calibration and comparing those estimates to the actual recharge that produced the calibration data.

\section{Estimating Recharge by Modeling Water Table Elevation}

In this section, we model the water table profile using the one-dimensional nonlinear Boussinesq equation of the form:

$$
\frac{K}{2} \frac{\partial^{2} h^{2}}{\partial x^{2}}+w(t)=S_{\mathrm{y}} \frac{\partial h}{\partial t}
$$

where $K$ is hydraulic conductivity, $w(t)$ is time-varying ground water recharge, $S_{\mathrm{y}}$ is specific yield, $x$ is position, $t$ is time, and $h$ is the water table elevation above the base of the aquifer (see, for example, Wang and Anderson 1982). We solve Equation 17 using a control volume finite-difference method (Patankar 1980). Nonlinearities were resolved iteratively.

We use the numerical model to compare transient and steady-state water table elevations. Specifically, we assign a time-varying recharge, $w(t)$, a priori and use this time series to calculate water table elevations, $h(x, t)$. We then calibrate a steady-state ground water flow model to selected values of $h(x, t)$, thereby determining the equivalent steady-state recharge rate $\left(w_{\mathrm{SS}}\right)$ that produces the same water table profile as the transient model at a given time. Finally, we compare $w_{\mathrm{SS}}$ and $w(t)$.
In these numerical experiments, we simulate water table elevations in a 305 -m-wide $(L=152.5 \mathrm{~m})$ onedimensional aquifer using a hydraulic conductivity of $3.05 \mathrm{~m} / \mathrm{d}$, a specific yield of $20 \%$, and constant-head boundary conditions specified at $3.05 \mathrm{~m}$ at both ends of the aquifer (Figure 1). We calibrate the steady-state model to the water table elevation at the center of the model (the point most sensitive to water table variation and, therefore, the optimal location for calibration).

\section{Periodic Recharge}

To a first approximation, ground water recharge is often periodic with a period of 1 year: in general, recharge tends to be high in the late autumn, winter, and early spring due to decreased evapotranspiration and tends to be low in the late spring, summer, and early autumn due to increased interception and evapotranspiration. With this in mind, we conduct a numerical experiment in which we impose a periodic recharge function, $w(t)=A \cos (b t)$, where $A=0.10 \mathrm{~cm} / \mathrm{d}, b=365 / \mathrm{d}$, and $t$ has units of days. The amplitude of the recharge function is roughly based on values of recharge estimated for coastal aquifers of the eastern United States (Anderson 1999), but the magnitude has no consequence on the conclusions we draw from these simulations. In the lower panel of Figure 2, we present a graph of specified timevarying recharge (solid line) that produces the water table hydrograph in the upper panel (solid line). We then sample this hydrograph and determine equivalent steadystate recharge rates by calibrating a numerical model of Equation 17. That is, Figure 2 shows temporal recharge variations for a single transient simulation (solid line, lower panel) and 138 steady-state simulations (circles, lower panel), which we determine by calibrating a
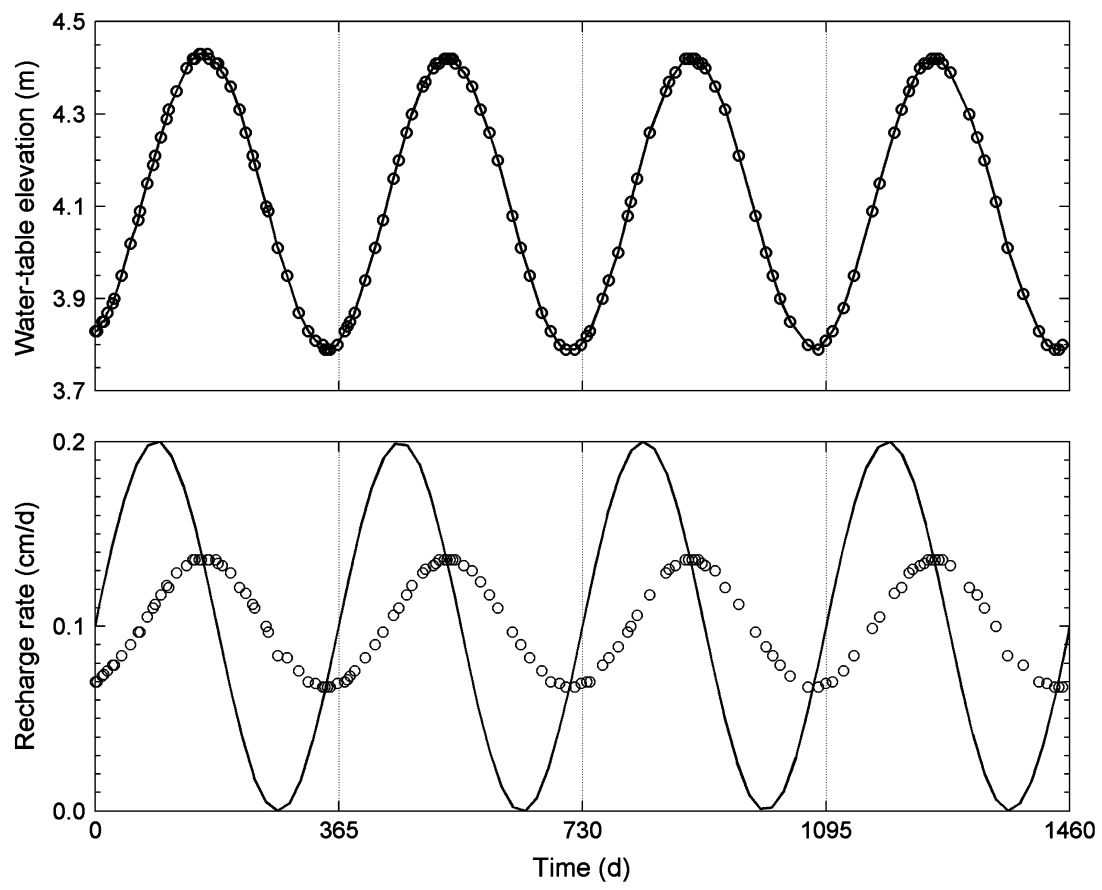

Figure 2. The lower panel shows periodic recharge (solid line) and steady-state recharge (circles) that produce the same water table profiles at a given time. The upper panel shows the water table hydrograph (solid line) generated by the periodic recharge and sampling points for steady-state recharge calibration (circles). 
steady-state model to water levels (solid line, upper panel) sampled at selected times (circles, upper panel).

Not surprisingly, the inferences we draw are identical to those in the previous section: namely, (1) when the water table is at a maximum or minimum, the steadystate recharge is equivalent to the instantaneous transient recharge; (2) during rising water table conditions, $w_{\mathrm{SS}}$ underestimates $w(t)$, and during falling water table conditions, $w_{\mathrm{SS}}$ overestimates $w(t)$; and (3) extreme values of steady-state recharge are less than extreme values of the equivalent transient recharge.

These model parameters were chosen somewhat arbitrarily, but the actual values do not affect the general conclusions we draw from the simulations. While the lag time between the instantaneous and steady-state recharge rates varies with transmissivity, the general rules that we mathematically and numerically demonstrated previously hold true no matter the transmissivity.

\section{Arbitrary Seasonal Recharge}

In the next numerical experiment, we extend our recharge calculations to the case in which transient recharge is not strictly periodic. In these simulations, we specify transient recharge as indicated by the solid line in the lower panel of Figure 3. These values of recharge are based on a 7-d moving average of precipitation in coastal North Carolina, United States ((Anderson 1999; Anderson et al. 2000). The averaged precipitation values are decreased to $30 \%$ of precipitation to account for runoff, interception, evaporation, and uptake by plants. We then fit a cubic spline to the data to get recharge rates at uniform time steps, which shifted the resulting curve downward and provided some negative recharge values that account for evaporative pumping by plants. The specific values and duration of recharge events do not affect the general conclusions that we draw from these simulations. The circles in the lower panel of Figure 3 show the equivalent steady-state recharge as determined by calibrating 72 separate steady-state simulations to water levels sampled (circles, upper panel) from a single transient simulation (solid line, upper panel). An examination of Figure 3 reveals that the inferences we made in the previous section apply generally and at all timescales.

\section{Calibrating to Average Water Levels}

One is tempted to assume that a steady-state model calibrated to long-term averages yields a long-term average recharge value. This is, in fact, the case provided water levels are averaged over very long times. In a previous section, we presented calculations to show that average water levels can indeed be used to estimate the actual average recharge rate provided that:

$$
t \gg S_{\mathrm{y}} L^{2} / T
$$

where $L$ is the half-width of the one-dimensional aquifer. In our final numerical experiment, we will demonstrate that this is indeed the case.

Consider the conceptual aquifer that was simulated in the previous examples. Using the aquifer's assigned physical parameters and the previous equation, we expect that average water levels can be used to estimate the average recharge rate as long as water levels are measured for much greater than 500 d. The plot shown in Figure 4, which uses the simulations of the previous numerical experiment, supports this conclusion.
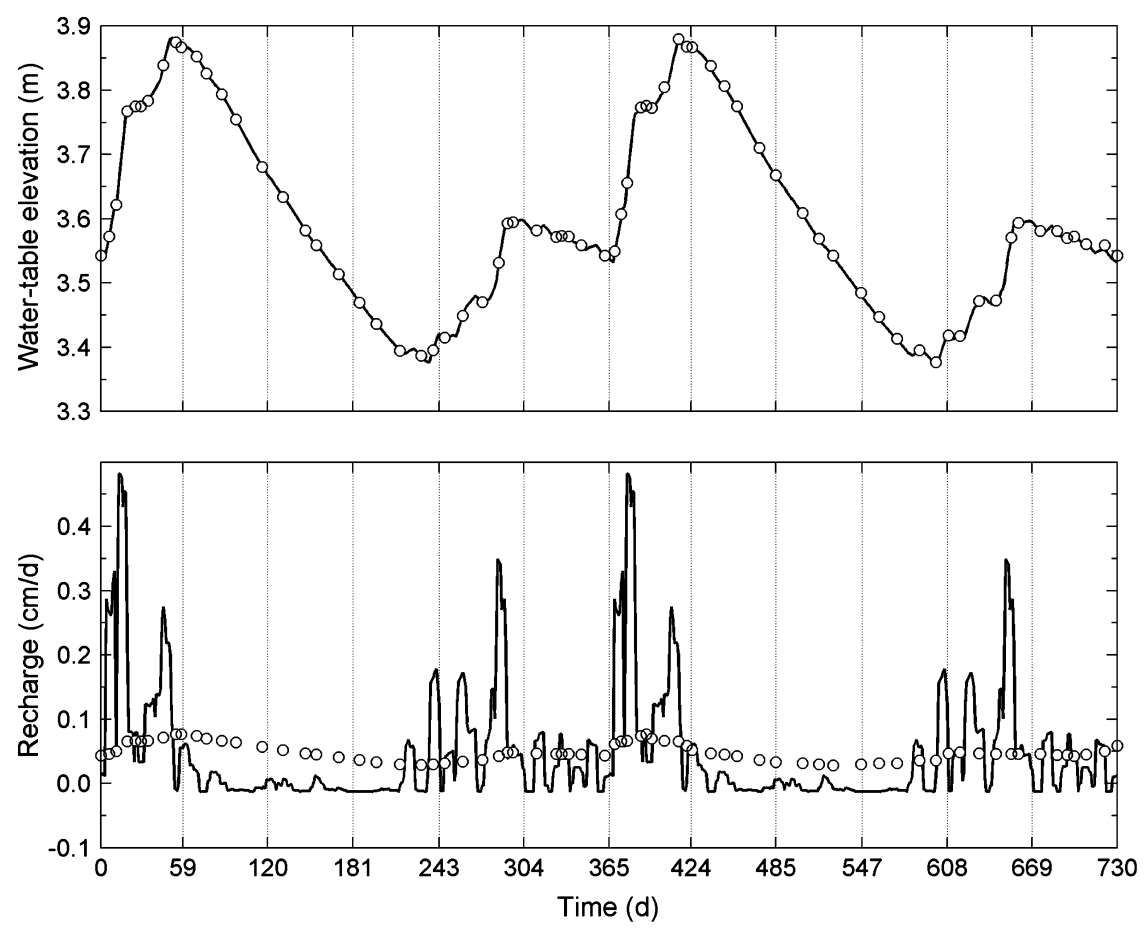

Figure 3. The lower panel shows a graph of recharge based on average precipitation data (solid line) and steady-state recharge (circles) that produce the same water table profiles at a given time. The upper panel shows a water table hydrograph (solid lines) and sampled values (circles) to which the steady-state simulations have been calibrated. 

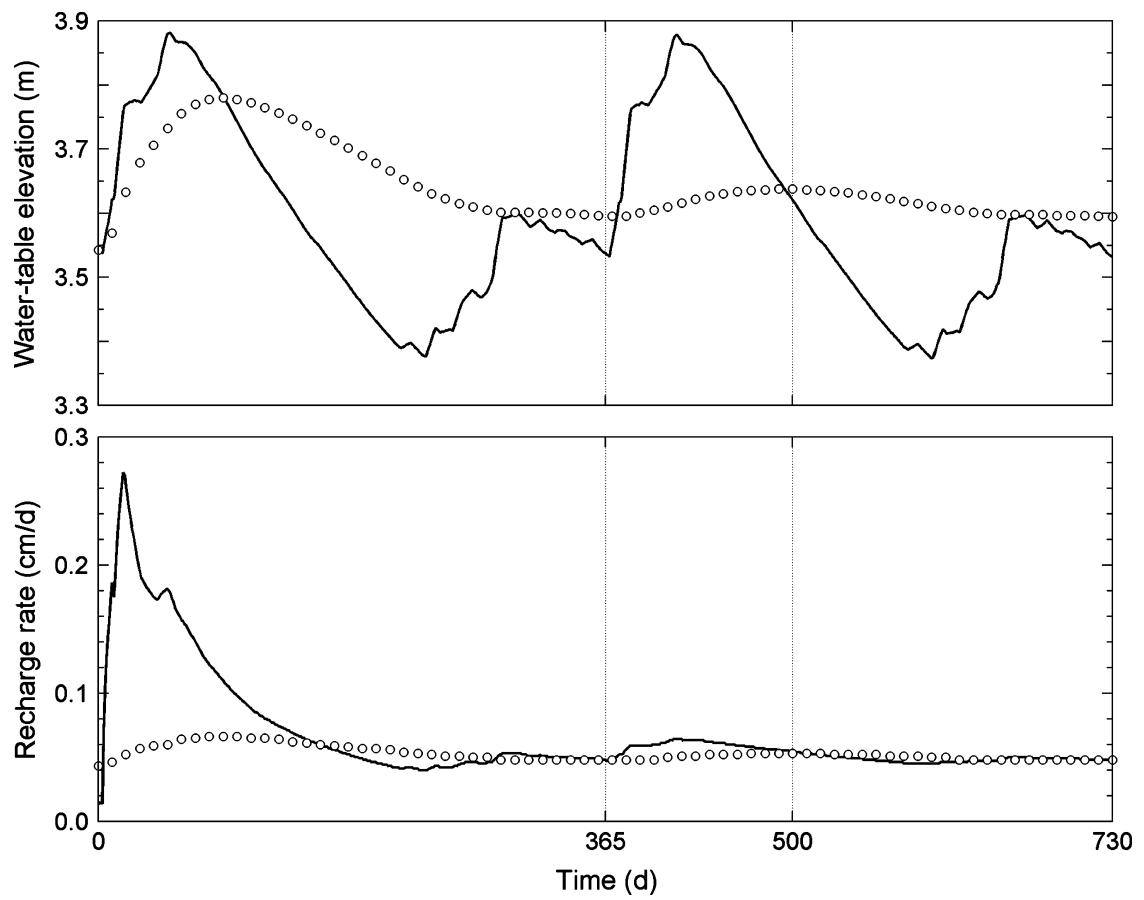

Figure 4. The running average of the transient recharge signal (solid line, lower panel) and steady-state recharge rates (circles, lower panel) calibrated to the running average of the water table hydrograph (circles, upper panel). The solid line on the upper panel is the water table hydrograph generated by the transient recharge signal.

Figure 4 shows the running average of the transient recharge signal of Figure 3 (solid line, lower panel) and steady-state recharge rates (circles, lower panel) calibrated to the running average of the water table hydrograph (circles, upper panel). The solid line in the upper panel is the water table hydrograph from Figure 3. The plot demonstrates that with sufficient time (beyond $500 \mathrm{~d}$ ), both the average transient recharge signal and the calibrated steady-state recharge rates begin to mimic each other. Fluctuations in water levels have a diminishing influence on the running average of the water level, which stabilizes to a level of $3.59 \mathrm{~m}$. Likewise, the behavior of the transient recharge function stabilizes to a recharge rate of $0.048 \mathrm{~cm} / \mathrm{d}$. We can further support our argument with a steady-state ground water flow simulation using a steady recharge rate of $0.048 \mathrm{~cm} / \mathrm{d}$ : the water table elevation predicted for the center of the aquifer with this steady-state recharge rate is $3.59 \mathrm{~m}$.

\section{Concluding Remarks}

There are several interesting implications of the results presented in this commentary. First, the mathematical considerations that we describe are not affected by varying the homogeneous hydraulic conductivity. Although the general rules that we present hold for all values of hydraulic conductivity, the lag time between the peak transient recharge and the subsequent peak steady-state recharge increases with decreasing hydraulic conductivity. Second, the mathematical considerations that we describe are not affected by incorporating spatially variable hydraulic conductivity and specific yield, nor are they affected by incorporating temporally variable and nonperiodic recharge functions. Therefore, the conclusions we draw would apply to specific regions of a spatially variable aquifer receiving a transient recharge signal. Third, in order for the steady-state recharge to equal the average recharge, we must have water level measurements accurately reflect the average water

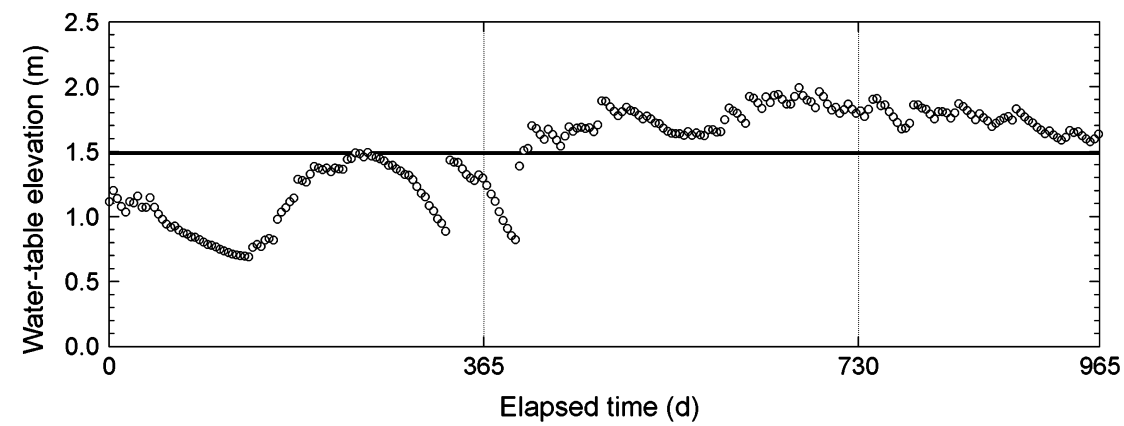

Figure 5. A graph of water table elevations (open circles) and average water levels (solid line) measured in a monitoring well near the center of Hatteras Island, North Carolina. 
level in the aquifer. Consider Figure 5, which shows water table fluctuations (open circles) and average conditions (solid line) measured in a monitoring well located near the center of Hatteras Island, North Carolina. Water level measurements that are biased to low water levels (e.g., measurements collected primarily during the dry season or during the extended drought conditions early in the monitoring period) will underestimate the average recharge, while water level measurements biased to high water levels (e.g., those collected after storm events or during long-term wet conditions) will overestimate the average recharge.

In many circumstances, hydrogeologists have no choice but to use a steady-state model to simulate ground water flow. For example, if model calibration is based on only a few water level measurements, such as early in the monitoring period of Figure 5, there may not be sufficient information on temporal variability to justify transient simulations. There may also not be sufficient data to calibrate to average water table elevations. Nonetheless, if recharge is used as a calibration variable, the modeler should understand the implications of using time-varying data to calibrate a steady-state model. We hope that this article provides some insight in this regard.

\section{Acknowledgments}

We would like to thank Jeff Raffensperger, Tom Burbey, Miroslav Nastev, and several anonymous reviewers whose insightful comments improved earlier versions of this manuscript. We would also like to thank the Thomas F. and Kate M. Jeffress Memorial Trust for providing funding for a portion of this study.

\section{References}

Anderson, M.P., and W.W. Woessner. 1992. Applied Groundwater Modeling: Simulation of Flow and Advective Transport. San Diego, California: Academic Press.
Anderson, W.P. Jr. 1999. The hydrology of Hatteras Island, North Carolina. Ph.D. diss., Department of Marine, Earth, and Atmospheric Sciences, North Carolina State University, Raleigh.

Anderson, W.P. Jr., D.G. Evans, and S.W. Snyder. 2000. The effects of Holocene barrier-island evolution on watertable elevations, Hatteras Island, North Carolina, USA. Hydrogeology Journal 8, no. 4: 390-404.

Ayers, J.F., and H.L. Vacher. 1983. A numerical model describing unsteady flow in a fresh water lens. Water Resources Bulletin 19, no. 5: 785-792.

Gelhar, L.W. 1974. Stochastic analysis of phreatic aquifers. Water Resources Research 10, no. 3: 539-545.

Haitjema, H.M. 1995. Analytic Element Modeling of Groundwater Flow. San Diego, California: Academic Press.

Hanson, R.T., M.W. Newhouse, and M.D. Dettinger. 2004. A methodology to assess relations between climatic variability and variations in hydrologic time series in the southwestern United States. Journal of Hydrology 287, 252-269, doi:10.1016/j.jhydrol.2003.10.006.

Kim, K., M.P. Anderson, and C.J. Bowser. 1999. Model calibration with multiple targets: A case study. Ground Water 37, no. 3: 345-351.

Manga, M. 1997. A model for discharge in spring-dominated streams and implications for the transmissivity and recharge of quaternary volcanics in the Oregon Cascades. Water Resources Research 33, no. 8: 1813-1822.

Manga, M. 1996. Hydrology of spring-dominated springs in the Oregon Cascades. Water Resources Research 32, no. 8: 2435-2439.

Patankar, S.V. 1980. Numerical Heat Transfer and Fluid Flow. New York: Hemisphere Publishing Corp.

Swanson, S.K., and J.M. Bahr. 2004. Analytical and numerical models to explain steady rates of spring flow. Ground Water 42, no. 5: 747-759.

Townley, L.R. 1995. The response of aquifers to periodic forcing. Advances in Water Resources 18, no. 3: 125-146.

Wang, H.F., and M.P. Anderson. 1982. Introduction to Groundwater Modeling. San Diego, California: Academic Press.

Zhang, Y.-K., and Z. Li. 2005. Temporal scaling of hydraulic head fluctuations: Nonstationary spectral analyses and numerical simulations. Water Resources Research 41, no. 7: W07031, doi:10.1029/2004WR003797. 